\title{
Exploration of Application of Computer Technology in E-commerce Network Consumption Interaction Xiaoxia Li
}

\author{
Shaanxi Institute of Technology, Xi'an, 710302m China
}

Keywords: computer technology; e-commerce network consumption; interaction application

\begin{abstract}
: particular mode expansion of e-commerce is closely related to electronic technology in network architecture. However, such dependence highlights potential security difficulties of e-commerce and hinders commercial progress for a long time. The peculiar technological path of computer is expanding continuously. Network consumption related to e-commerce also integrates microcomputer technology under new situations and promotes commercial extension. Multiple data gained from network marketing shall be classified. It is necessary to deeply analyze this interactive background under the planned demand. Network consumption within the e-commerce category creates of interaction and covers diversified behaviors in daily life.

In e-commerce expansion, microcomputer technology synergy highlights the prominent value. The common application of microcomputer technology boots security assurance within the network category. Under new situations, new channels are preset for e-commerce, and the marketing amount is reduced. Meanwhile, the quantity of consumption also increases progressively. Relying on computer, marketing approaches of e-commerce highlight the tendency of efficiency. Merchants in business category can make electronic boards, send e-mail, and enter the set website to deliver every-changing commercial information. In this way, original interactive effect is limited and reduced. Depending on websites, enterprises can clearly know diversified information in each time bucket, cut down accumulative inventory and create the optimal interaction effect.
\end{abstract}

\section{I. particular technical advantage}

(I) E-shop

Compared with physical stores in traditional situation, e-shops which have been accepted gradually decrease site selection, follow-up store decoration and personnel recruitment and cut down consumed time and money. New services created by e-shops highlight distinct individuality. Low consumption of e-shops will also promote decrease of selling price. Real-time information exchange can help construct convenient and fast exchange paths. Sufficient resources on network facilitate advertising and cut down original cost of advertising. Therefore, construction of e-shops cannot be separated from microcomputer.

E-commerce involves marketing in network architecture, set electronic accounts, negotiation with publicity characteristic, ordering under network synergy, network path payment, matched logistic service and useful opinions collected. Multi-level consumption functions will depend on microcomputer. Microcomputer ensures security for online consumption of e-commerce.

(II) Supply of automatic service

Multi-level functions preset by e-commerce include wider category. It integrates shopping online, online payment and network control with security characteristic. Diversified services within automation category tally with consumer psychology. The subdivided functions include the following levels:

Firstly, network ordering. The set mode of e-commerce supports online ordering. When consumers check a network preset, they can draft e-mails and purchase articles online. In the set purchase order, consumers confirm goods information and create convenient and fast ordering. Due to the characteristic of online payment, buyers and sellers pay online according to credit card with electronic property (i.e. e-bank).

Secondly, e-commerce expands publicity and covers a wider range of advertisement. Consumers check useful information via network. Meanwhile, merchants expand original publicity range by 
virtue of webpage and drafted emails. In such case, wider range of consumers can clearly know unique advantages of goods and are pleased to buy them.

Thirdly, e-commerce together with merchants manages the network. It integrates enterprises and users. For subdivided customer categories, it will identify orderly and add management.

\section{Correlation between commerce and new technology}

Unique advantages of microcomputer technology mostly stand out in e-commerce of new time bucket. E-commerce owns multiple properties. It is a trading market under virtual situation. By such commerce, people can settle space-time barrier and directly communicate. Enterprise purchase and marketing step setting in the initial stage will often result in much cost. Under network architecture, commercial framework with virtual property reduces consumption expenditure. Diversified trading with bilateral property is included in macroscopic network. It displays promotion function for economic progress in the region.

Under the support of communication, e-commerce with modern property provides network operation. Unique superiority of microcomputer is timely information communication. The expense used to eliminate space-time barrier and add initially is not high, either. Irreplaceable support of e-commerce covers computer. The optimal marketing path integrates microcomputer technology, electronic technique with network synergy and virtual commerce with electronic property. Such new situation expands application region of commercial framework and also provides safety curtain for commercial expansion in a long time. This facilitates long-term development at the original level.

\section{Real application of interaction path}

(I) To create virtual goods

The virtual shopping mall constructed by the elements e-commerce covers is the consumption prediction in the network framework. Goods in the shopping mall are diversified goods with virtual property. In such situation, the computer should be adopted to prepare such gods with virtual property. The general environment e-commerce established by e-commerce will not be limited, and space-time barrier is removed. During making these goods with virtual property by microcomputer, multi-level consumption suggestions can be collected. Such goods will tally with wider range of demands. After the consumption demands are clearly known, new products can be added. This can improve interaction convenience under the general situation.

(II) To bring convenience for real-time communication

If consumers clear know their demands, they can collect all goods complying with their demands through search tool preset on network. This reduces browsing time and provides multiple conveniences. Such services under new situation have obvious personality trend and highlight pertinence. Aiming at a product screed out, the merchant can expand the original advertisement range so as to improve original popularity. The advertisement on network can cut down expenditure and covers sufficient information. This provides the best path for continuous market expansion. Information interaction under immediate state highlights high-level convenient and fast advantage. It breaks through usual mode limitation and achieves immediate interaction.

(III) To manage shopping mall orderly

Computer technology can create management approaches with actual effect property and properly manage the shopping mall established. The number of existing shopping malls is on progressive increase. In such tendency, beautification link of the shopping mall can assist merchants in standing out rapidly. Art design in the network framework can add original attraction of the shopping mall, increase page view in each time bucket and promote the progressive increase in the sum of consumption. The novel operation mode is adopted to check information prudently so as to ensure information accuracy. This improves original consumption quality and further constructs the platform interactions repay on. 


\section{Maintain interaction safety}

The preset thought of e-commerce integrates microcomputer technology in the new time bucket and communication in the network category. Double techniques under the architecture cannot be separated from original microcomputer technology. Online consumption which e-commerce depends on is closely related to internet in the section. However, there are many potential threats in network. For example, interaction information is replaced, tampered, damaged or illegally stolen in the transaction. Some lawbreakers even simulate and change original real information so that transaction is interrupted. Transaction safety can be ensured in each time bucket only through new technology.

(I) Secret key encryption

Data encryption approaches such as matched secret key can be adopted to construct network encryption. In the consumption process, private information includes set consumption account, matched password and payment password. If the information is lost, larger loss will be caused. Such potential danger highlights the significant value of encryption. Usual encryption mode is PKI technology. It presents secret key and guarantees multiple information safety in each transaction time bucket.

Under the peculiar technological path of PKI, the secret key which is set well is divided into a pair of refined secret keys. One secret key can be set to be open, while proper decryption should be added for the other one and properly kept. In this way, private information can be added through the open secret key. Meanwhile, the other secret key can be used for convenient decryption. The secret key under open state can be issued in a larger category, but the corresponding one under exclusion situation can be only deemed as the preset and generated secret key for the related party. This maintains transaction safety in multiple links.

(II) Signature with anti-fake property

Digital signature held in consumption transaction can avoid usual fake. The open secret key can be used to add encryption approach for necessary information screed out. Such technology owns derivation characteristic. Digital signature anti-fake depends on constrains transaction subjects and avoid denial of related information or vicious manipulation.

(III) Identification of real identity

The technology required by identity identification tallies with unique attribute of e-commerce. Compared with transaction approach under traditional architecture, transaction parties in the category of e-malls do not need to meet each other. Such convenient and fast approach reduces time consumption, but meanwhile increases potential risks.

Advance technology of identity identification can distinguish corresponding transaction subjects. This cuts down errors and promotes identity accuracy. The proper identity identification technology can also be used to expand in other fields. Unique identification technique of intelligent card reduces potential illegal identity and makes consumers in the network far from hacker attack.

(IV) Resistance of illegal invasion

Intelligent firewall can get rid of hidden virus and hinder illegal access and invasion. The new fire wall omits tedious operations. At present, the number of network viruses is on progressive increase and miscellaneous types appear. Technical attribute of virus is also promoting continuously. Firewall under intelligent architecture provides clear direction for continuous technical development.

In recent years, network expansion sped increases. Correspondingly, virus transmission paths are also increasing continuously. The transmission speed of virus and corresponding expansion level highlight the rising trend. It is very hard to gain good effects to control such viruses by single computer, so, it is required to establish LAN and create virus control under 3D situation. The set gateway or exclusive prevention and control software is adopted to hinder such invasion in the special time buckets of network consumption. For instance, when email is adopted, matched prevention and control software shall be added to identify the connotation covered by the software. 


\section{Construct brand emphasis}

(I) To highlight distinctive differences

Difference attribute reflected in e-commerce is highlighted in the goods. New brands can highlight differences. Merchants put particular emphasis on seeking peculiar services and unique connotation of goods. If new brands need to be created under independent situation, unique brand characteristics of rivals should be clearly known. In addition, if such differences are not highlighted in goods, clear differences may be highlighted in the follow-up selling process so as to attract consumption.

For instance, name card holders of a brand are usually packed for selling. In the novel selling approach, the four single products are placed in the same package. For example, full sets of household articles are arranged in the small stores for household articles so as to highlight single product. Such marketing thought highlights unique trend and can easily attract users. Merchants can gain profits only through adding novel characteristics in usual marketing paths.

(II) To create unique thought

Under network consumption situation, some dealers just focus on replacing good labels, and cannot create novel private brands. In the research and development period, unique attributes shall be represented. Only unique goods can create high-level selling value and construct long-term consumption correlation.

(III) To pay attention to interaction experience

Under the peculiar interaction path of network consumption, relevance should be constructed for marketing ideas and preset objects. Such relevance may be represented in unique shopping experience, or in a type of goods. Relevance can also be constructed for the brands in similar category. Communication and exchange should pay attention to unique experience.

For instance, differences may be highlighted in customers' experience for subdivided goods types. It is required to check consumption information fed back in time and identify such differences. The daily services should have consistence. High-quality services under the same situation should be supplied for various users. This can boost sense of value of the brand and improve management level.

\section{Conclusions}

Rapid development of microcomputer network facilitates promotes commercial progress under interaction path. The data saved in the computer ensures interaction safety in each time bucket through encryption, identity identification, and digital signature with digital characteristic. According to consu8motion suggestions gained from feedbacks, goods types under virtual situation can be added anytime. Under microcomputer synergy, it is necessary to construct commercial website, orderly manage this website, reduce advertisement cost and provide guarantee for interaction progress in future period.

\section{References}

[1] Jia Lifei, On functions of computer technology in e-commerce network consumption interaction [J]. Oriental Enterprise Culture, 2014 (03), 23:228

[2] Pan Tan, Functions of computer technology in e-commerce network consumption interaction [J]. Guide to Business, 2014 (06), 23:42+41

[3] Zhang Lei, Functions of computer technology in e-commerce network consumption interaction [J]. Value Engineering, 2014 (08), 03:190-191

[4] Wang Dandan, Functions of computer technology in e-commerce network consumption interaction [J]. Computer CD Software and Application, 2014 (04), 17:45-46

[5] Lv Yunfang, Exploration of functions in computer technology in e-commerce [J]. Silicon Valley, $2012(07), 18: 30+5$ 
